\title{
Outcomes of patients with borderline resectable and resectable pancreatic adenocarcinoma treated with neoadjuvant three-week course chemoradiotherapy using capecitabine-based versus gemcitabine-based concurrent chemotherapy
}

\author{
Shane S. Neibart ${ }^{1}$, Swati Mamidanna ${ }^{1}$, Anupama Chundury ${ }^{1}$, Mutlay Sayan ${ }^{2}$, H. Richard Alexander ${ }^{3}$, \\ David A. August ${ }^{3}$, Lyudmyla D. Berim ${ }^{4}$, Patrick M. Boland ${ }^{4}$, Miral S. Grandhi ${ }^{3}$, Prateek Gulhati ${ }^{4}$, \\ Howard S. Hochster ${ }^{4}$, Russell C. Langan ${ }^{3}$, Kristen R. Spencer ${ }^{4}$, Timothy J. Kennedy ${ }^{3}$, Matthew P. Deek ${ }^{1}$, \\ Salma K. Jabbour ${ }^{1}$ \\ ${ }^{1}$ Department of Radiation Oncology, Rutgers Cancer Institute of New Jersey, New Brunswick, NJ, USA; ${ }^{2}$ Department of Radiation Oncology, Dana- \\ Farber Cancer Institute, Brigham and Women's Hospital, Harvard Medical School, Boston, MA, USA; ${ }^{3}$ Division of Surgical Oncology, Rutgers \\ Cancer Institute of New Jersey, New Brunswick, NJ, USA; ${ }^{4}$ Division of Medical Oncology, Rutgers Cancer Institute of New Jersey, New Brunswick, \\ NJ, USA \\ Contributions: (I) Conception and design: SS Neibart, SK Jabbour; (II) Administrative support: SS Neibart, SK Jabbour; (III) Provision of study \\ materials or patients: A Chundury, M Sayan, HR Alexander, DA August, LD Berim, PM Boland, MS Grandhi, P Gulhati, HS Hochster, RC Langan, \\ KR Spencer, TJ Kennedy, SK Jabbour; (IV) Collection and assembly of data: SS Neibart, S Mamidanna; (V) Data analysis and interpretation: SS \\ Neibart, S Mamidanna, A Chundury, SK Jabbour; (VI) Manuscript writing: All authors; (VII) Final approval of manuscript: All authors. \\ Correspondence to: Salma K. Jabbour, MD. Department of Radiation Oncology, Rutgers Cancer Institute of New Jersey, New Brunswick, NJ 08901, USA. \\ Email: jabbousk@cinj.rutgers.edu.
}

Background: Neoadjuvant chemoradiotherapy can provide downstaging and improve margin negativity for borderline resectable and resectable pancreatic adenocarcinoma [(B)RPC]. Little is known about the relative efficacy of capecitabine (CAPE)-based vs. gemcitabine (GEM)-based 3-week chemoradiation (3WCRT) with 36 Gy in 15 fractions. This study aimed to compare the odds of achieving surgical resection, time to progression (TTP), and overall survival (OS) of patients treated with 3 WCRT with concurrent CAPE versus GEM.

Methods: A retrospective cohort study was conducted, examining medical records from a single center for patients with (B)RPC treated with 3WCRT between 1/2009-12/2020. Odd ratios (OR) of achieving surgical resection were estimated using logistic regression for univariable and multivariable analyses. Median TTP (mTTP) and median OS (mOS) were estimated using the Kaplan-Meier method. Cox proportional hazards analysis was conducted to estimate hazard ratios (HR) of progression and survival in univariable and multivariable analyses.

Results: Thirty-one patients were included in the analysis. Twenty-two (71\%) patients were treated with CAPE, while 9 (29\%) were treated with GEM. All patients in the GEM group were borderline resectable, vs. $18(82 \%)$ patients in the CAPE group, $\mathrm{P}=0.30$. Nineteen $(86 \%)$ patients in the CAPE group were treated with neoadjuvant FOLFIRINOX, vs. 4 (44\%) patients in the GEM group, $\mathrm{P}=0.03$. The CAPE group had higher odds of achieving surgical resection [OR =9.33; 95\% confidence interval (CI): 1.50-58.20]. Adjusting for covariates, the odds of achieving surgical resection were still statistically higher in the CAPE group vs. the GEM group (OR =25.34; 95\% CI: 1.14-563.72). The CAPE group had superior mTTP compared to the GEM group (15.4 months, 95\% CI: 4.9-71.1 vs. 4.0 months, 95\% CI: 0.4-14.5; P=0.01), corresponding to a hazard ratio of 0.33 (95\% CI: 0.14-0.81). Adjusting for covariates this effect persisted; the adjusted hazard ratio (AHR) for progression was 0.24 (95\% CI: 0.08-0.77). Cox proportional hazards analysis also demonstrated that the CAPE group had superior OS compared to the GEM group in unadjusted (HR $=0.13$; 95\% CI: 0.04-0.40) and adjusted models (HR =0.13, 95\% CI: 0.03-0.52).

(C) Journal of Gastrointestinal Oncology. All rights reserved. 
Conclusions: For neoadjuvant 3WCRT, this hypothesis-generating study suggests concurrent CAPE may be a more effective radiosensitizer than GEM for patients with (B)RPC.

Keywords: Pancreatic cancer; neoadjuvant; chemoradiation; capecitabine (CAPE); gemcitabine (GEM)

Submitted Aug 19, 2021. Accepted for publication Sep 24, 2021.

doi: 10.21037/jgo-21-503

View this article at: https://dx.doi.org/10.21037/jgo-21-503

\section{Introduction}

Pancreatic ductal adenocarcinoma (PDAC) represents just $3 \%$ of all cancer diagnoses annually but is the fourth leading cause of cancer-related death in the United States (1). Approximately 15-20\% of all PDAC diagnoses are borderline resectable or resectable at diagnosis, typically necessitating neoadjuvant treatment to enable downstaging and margin-negative resection (2-6). Margin-negative resection is strongly associated with survival, but the optimal preoperative regimen to facilitate an optimal surgical outcome is highly debated (7).

A 3 -week course of chemoradiation (3WCRT) with 3,600 cGy in 15 fractions is a regimen utilized for patients with borderline resectable and resectable pancreatic cancer [(B)RPC] (8). First evaluated in patients with locally advanced PDAC, 3 WCRT with concurrent gemcitabine (GEM) was extended to the (B)RPC setting after demonstrating promising surgical and survival outcomes, even for patients who were deemed initially unresectable (9-12). The preoperative radiochemotherapy versus immediate surgery for resectable and borderline resectable pancreatic cancer (PREOPANC)- 1 trial subsequently demonstrated advantages of a neoadjuvant approach with 3 WCRT over directly proceeding with surgery (13). Although the study failed to show an overall survival (OS) benefit of 3 WCRT with concurrent GEM, the 3 WCRT group had superior R0 resection rate, diseasefree survival, and locoregional control. The PREOPANC trial utilized concurrent GEM in their 3WCRT strategy, but there is evidence from the Selective Chemoradiation in Advanced Localised Pancreatic Cancer (SCALOP) trial, which tested a longer course of chemoradiation $(5,040$ cGy in 28 fractions), that concurrent capecitabine (CAPE) is the optimal concurrent chemotherapy for neoadjuvant chemoradiation when compared to concurrent GEM (14). We hypothesize that the benefits of concurrent CAPE over concurrent GEM will also translate to 3WCRT. The primary objectives of the study are to evaluate the odds of achieving surgical resection and time to progression (TTP) for patients treated with 3WCRT with concurrent CAPE (CAPE group) or concurrent GEM (GEM group). We also aim to explore the patterns of progression and OS of patients treated with the aforementioned regimens.

We present the following article in accordance with the STROBE reporting checklist (available at https://dx.doi. org/10.21037/jgo-21-503).

\section{Methods}

\section{Data source and cohort definition}

Electronic health records were retrospectively reviewed at a National Cancer Institute-Designated Comprehensive Cancer Center for patients diagnosed with PDAC between January 1st, 2009 and December 31st, 2020. All patients with clinical notes from surgical oncologists dictating that they were borderline resectable or resectable at time of diagnosis were included. Patients treated with neoadjuvant 3WCRT with either concurrent GEM or CAPE were included in the analysis.

\section{Measures}

Concurrent chemotherapy was determined by assessing radiation oncology and medical oncology progress notes. The GEM group was defined as any patient starting 3WCRT with concurrent gemcitabine (weekly infusions). Similarly, the CAPE group was defined as patients starting 3 WCRT with concurrent capecitabine (twice-per-day dosing). All patients underwent computed tomography (CT) simulation with a large rigid pillow for immobilization with intravenous and oral contrast. Four-dimensional (4D)CT simulation was available as of 2011. Either an intensity modulated, or 3D-conformal radiation therapy (RT) technique was employed. Volumes included the gross tumor volume including the primary tumor and detectable lymph nodes with a 1-2 cm expansion for the clinical target volume 
(CTV) as well as a CTV incorporating para-aortic lymph nodes at the level of the primary tumor, peripancreatic lymph nodes, the porta hepatis/portal venous confluence, and at-risk vessels (i.e., celiac artery, superior mesenteric artery and vein). A dose of 3,600 cGy in 15 fractions over 3 weeks Monday-Friday was delivered.

By assessing operative reports and progress notes, achieving surgical resection was defined as any patient treated with pancreaticoduodenectomy or distal pancreatectomy regardless of organ-sparing approach. Pathology records were reviewed to determine margin status and evidence of downstaging with preoperative treatment. TTP was determined by assessing progress notes and radiology reports for evidence of progression. Date of last follow-up, and inpatient and outpatient records were also reviewed to determine censoring dates and dates of death to estimate OS. TTP and survival estimates were calculated from the start of 3WCRT.

Baseline characteristics (age at diagnosis, Eastern Cooperative Oncology Group Performance Status, gender, nodal status, year of diagnosis, weeks of neoadjuvant chemotherapy, neoadjuvant chemotherapy type, and borderline $v s$. resectable status) were collected at the time of diagnosis. Choice of neoadjuvant chemotherapy was classified as FOLFIRINOX or gemcitabine (with or without nab-paclitaxel). Additionally, CA 19-9 levels were assessed immediately before starting neoadjuvant chemotherapy and before starting 3 WCRT.

For describing the patterns of progression, location of failure was determined by reviewing interval imaging reports and clinical notes. Patterns of progression were characterized as local, regional, local and distant progressions.

\section{Statistics analysis}

Baseline characteristics were compared between CAPE and GEM groups. Continuous variables were described using median and interquartile ranges (IQR) and were compared using Wilcoxon-rank test. Categorical variables were described with frequencies and percentage and were compared using Fisher exact test. Odd ratios (OR) of achieving surgical resection were computed and presented with $95 \%$ confidence interval (CI) using logistic regression. Adjusted odd ratios (AOR) and 95\% CI were estimated. Median TTP (mTTP) and median OS (mOS) with 95\% CI were estimated using the Kaplan-Meier method; statistical significance was determined using the log-rank test. Cox proportional hazards analysis was conducted to estimate hazard ratios (HR) for progression and death with or without inclusion of confounding factors. Median followup time was determined using the reverse KaplanMeier method. Multivariable logistic regression and Cox proportional hazards models included age at diagnosis and any baseline characteristics with a $\mathrm{P}$ value less than 0.2 . Additional sensitivity analyses were conducted only analyzing progression and survival outcomes of patients treated with neoadjuvant FOLFIRINOX. All analyses were conducted with or without including resectable patients. All $\mathrm{P}$ values were two-sided and were compared to an alpha cut-off of 0.05. All statistics were computed using SAS 9.4 (SAS Institute, Cary, NC, USA).

\section{Ethical statement}

This study was conducted in accordance with the Declaration of Helsinki (as revised in 2013), and was approved by the Institutional Review Board of Rutgers Biomedical and Health Sciences (No. Pro2018002435), and individual consent for this retrospective analysis was waived.

\section{Results}

\section{Baseline characteristics}

There were 31 patients included in the analysis. Nine (29\%) patients were treated with GEM-based 3WCRT, and 22 (71\%) patients were treated with CAPE-based 3WCRT. All patients completed the 15 -fraction regimen. Baseline characteristics are included in Table 1. Of note, there was no statistically significant difference in age at diagnosis, gender, ECOG PS, weeks of neoadjuvant chemotherapy, node status, and CA 19-9 levels before chemotherapy and before 3WCRT. There were no patients in the GEM group that were deemed resectable at the time of diagnosis, but $4(18.2 \%)$ patients were resectable in the CAPE group, though this difference was not statistically significant. Choice of neoadjuvant chemotherapy was different between groups as $19(86 \%)$ patients were treated with neoadjuvant FOLFIRINOX in the CAPE group whereas only 4 (44\%) patients were treated with FOLFIRINOX in the GEM group $(\mathrm{P}=0.03)$. Among patients who were treated with neoadjuvant gemcitabine, $3(66 \%)$ and $2(60 \%)$ were also treated with nabpaclitaxel in the GEM and CAPE group, respectively. Four (44\%) and 1 (5\%) patient were treated between 2009-2016 in the GEM and CAPE groups, respectively $(\mathrm{P}=0.0171)$. The 
Table 1 Baseline characteristics of patients treated with CAPE-based vs. GEM-based concurrent chemoradiation

\begin{tabular}{|c|c|c|c|}
\hline Covariate & CAPE-based, $n=22, n$ [\%] & GEM-based, n=9, n [\%] & $P$ value \\
\hline \multicolumn{4}{|l|}{ Gender } \\
\hline Male & 13 [59.09] & $7[77.78]$ & 0.4290 \\
\hline Female & 9 [40.91] & 2 [22.22] & \\
\hline 0 & 9 [40.91] & 3 [33.33] & \\
\hline 1 & $12[54.55]$ & $5[55.56]$ & \\
\hline 2 & $1[4.55]$ & $1[11.11]$ & \\
\hline BR vs. R & & & 0.2952 \\
\hline Nodal status & & & 0.6125 \\
\hline 0 & 19 [86.36] & $7[77.78]$ & \\
\hline 1 & 3 [13.64] & 2 [22.22] & \\
\hline Year of diagnosis & & & 0.0171 \\
\hline 2009-2015 & $1[4.55]$ & 4 [44.44] & \\
\hline 2016-2020 & $21[95.45]$ & $5[55.55]$ & \\
\hline Pre-Chemo CA 19-9 & & & 0.2524 \\
\hline Below median & 13 [59.09] & 3 [33.33] & \\
\hline FOLFIRINOX & 19 [86.36] & $4[44.44]$ & \\
\hline GEM & 3 [13.64] & $5[55.56]$ & \\
\hline Weeks of NA Chemo*, weeks & $8.0[8.0-14.0]$ & 11.5 [10.0-18.0] & 0.1380 \\
\hline
\end{tabular}

*, median [interquartile range] presented. CAPE, capecitabine; GEM, gemcitabine; ECOG PS, Eastern Cooperative Oncology Group Performance Status; BR, borderline resectable; R, resectable; Chemo, chemotherapy; RT, radiation therapy; NA, neoadjuvant.

median weekly dose for the GEM group was $675 \mathrm{mg} / \mathrm{m}^{2}$ (IQR: 600-912.5). Two (9\%) patients in the CAPE group received twice daily dosing of $625 \mathrm{mg} / \mathrm{m}^{2}$, whereas the other $20(91 \%)$ received twice daily dosing of $825 \mathrm{mg} / \mathrm{m}^{2}$. There were no dose reductions or treatment breaks in either group. Year of diagnosis, weeks of neoadjuvant chemotherapy, and type of neoadjuvant chemotherapy all had $\mathrm{P}$ values below the pre-specified cut-off of 0.2 , so were included in multivariable models along with age at diagnosis. The median follow-up time was 29.4 months; there were 570.3 person-months of follow-up time.

\section{Achieving surgical resection}

Patients treated with CAPE-based 3WCRT had higher odds of achieving surgical resection than the GEM-based 
Table 2 OR for proceeding with surgery for patients treated with chemoradiation with concurrent CAPE or GEM

\begin{tabular}{lccccc}
\hline \multirow{2}{*}{ Concurrent Chemo } & \multicolumn{2}{c}{ Borderline resectable and resectable } & & \multicolumn{2}{c}{ Borderline resectable only } \\
\cline { 2 - 6 } & OR $(95 \% \mathrm{Cl})$ & AOR $(95 \% \mathrm{Cl})$ & OR $(95 \% \mathrm{Cl})$ & AOR $(95 \% \mathrm{Cl})$ \\
\hline GEM & Ref. & Ref. & Ref. & Ref. \\
CAPE & $9.33(1.50-58.20)$ & $24.75(1.21-505.13)$ & & $7.00(1.10-44.61)$ & $25.34(1.14-563.72)$ \\
\hline
\end{tabular}

AOR is adjusted by year of diagnosis, weeks of neoadjuvant chemotherapy, and type of neoadjuvant chemotherapy. OR, odd ratios; CAPE, capecitabine; GEM, gemcitabine; Chemo, chemotherapy; $\mathrm{Cl}$, confidence interval; AOR, adjusted odd ratios.
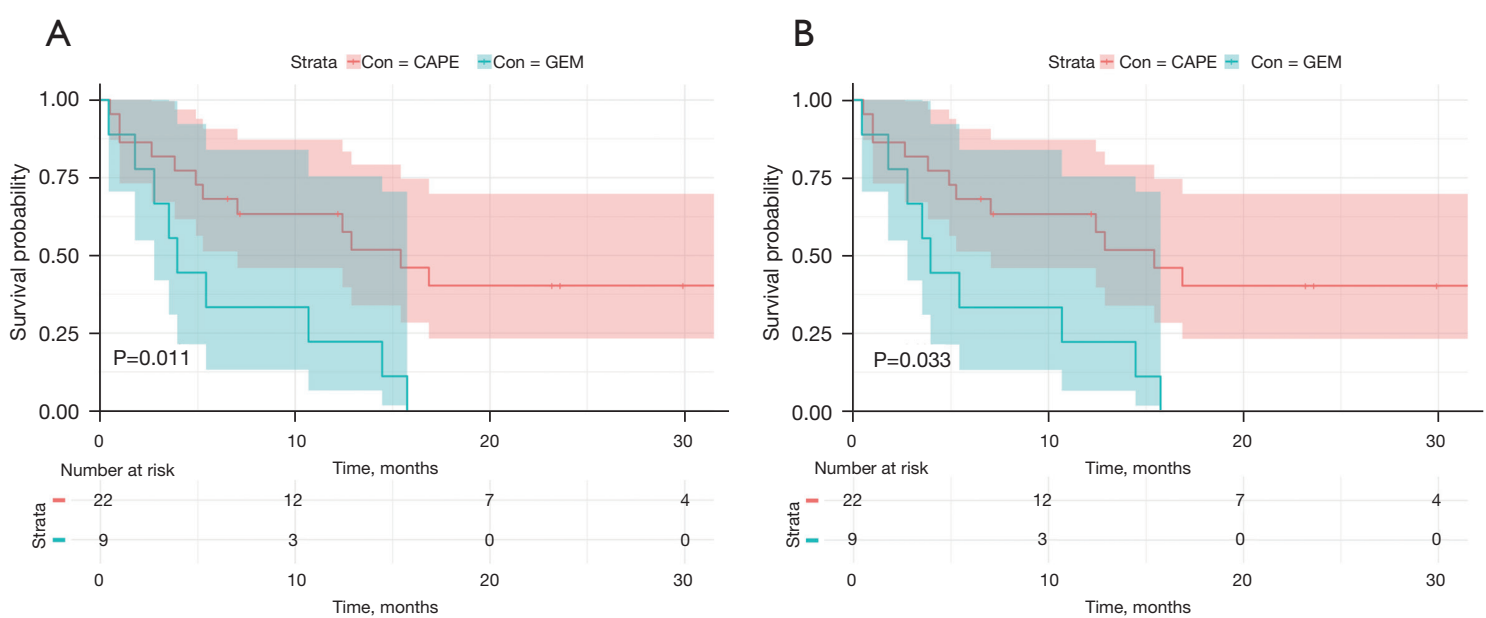

Figure 1 Kaplan-Meier plot of TTP for chemoradiation with concurrent CAPE vs. GEM with (A) or without (B) resectable patients included in analysis. TTP, time to progression; CAPE, capecitabine; GEM, gemcitabine.

group $(\mathrm{OR}=9.3 ; 95 \% \mathrm{CI}: 1.5-58.2)$. After excluding patients who were resectable at diagnosis $(n=4$, patients from the CAPE group), this effect was attenuated but still statistically significant $(\mathrm{OR}=7.0 ; 95 \% \mathrm{CI}: 1.1-44.6)$. Adjusting for covariates, the odds of achieving surgical resection were still statistically higher in the CAPE group $v s$. the GEM group (for borderline resectable and resectable: $\mathrm{OR}=24.8 ; 95 \%$ CI: $1.2-505.1$; only borderline resectable: $\mathrm{OR}=25.3 ; 95 \%$ CI: 1.1-563.7). These results are summarized in Table 2 . Among the patients who underwent resection, 2 (100\%) patients in the GEM group and 15 (93.75\%) patients in the CAPE group had negative margins. Among this same group, $1(50 \%)$ patient in the GEM group and $6(38 \%)$ in the CAPE group had tumor downstaging in response to neoadjuvant treatment.

\section{TTP}

Patients treated with CAPE-based 3WCRT had a statistically superior mTTP of 15.4 months (95\% CI:
4.9-71.1), whereas the GEM group had a mTTP of 4.0 months (95\% CI: $0.4-14.5$ ), $\mathrm{P}=0.01$ (Figure $1 A$ ). This difference corresponded to a hazard ratio of 0.33 (95\% CI: 0.14-0.81) (Table 3). After excluding the resectable patients from the CAPE group this effect persisted (mTTP of 12.9 months (95\% CI: 3.8-71.1; $\mathrm{P}=0.03$ ) (Figure $1 B$ ). This difference corresponded to a hazard ratio of 0.38 (95\% CI: 0.15-0.96) (Table 3). After adjusting for relevant covariates using multivariable Cox regression, the CAPE group had superior TTP than the GEM group (AHR $=0.24 ; 95 \%$ CI: 0.07-0.80). This effect was similar after excluding those with resectable pancreatic cancer at diagnosis (AHR $=0.24$; 95\% CI: 0.08-0.77).

Sensitivity analysis excluding patients who were not treated with neoadjuvant FOLIFIRINOX was also conducted and results were summarized in the Table S1. The unadjusted model (HR $=0.42 ; 95 \%$ CI: $0.13-1.35$ ) and adjusted model excluding patients with resectable disease (AHR $=0.35 ; 95 \%$ CI: 0.09-1.42) were similar to the models in Table 3, but CI widened. 
Table 3 HR of progression and survival for patients treated with chemoradiation with concurrent CAPE or GEM with or without resectable patients in analysis

\begin{tabular}{llllll}
\hline \multirow{2}{*}{ Outcome } & \multicolumn{2}{l}{ Borderline resectable and resectable } & & \multicolumn{2}{l}{ Borderline resectable only } \\
\cline { 2 - 3 } Progression & $\mathrm{HR}(95 \% \mathrm{Cl})$ & $\mathrm{AHR}(95 \% \mathrm{Cl})$ & $\mathrm{HR}(95 \% \mathrm{Cl})$ & $\mathrm{AHR}(95 \% \mathrm{Cl})$ \\
\hline Survival & $0.13(0.14-0.81)$ & $0.24(0.07-0.80)$ & $0.38(0.15-0.96)$ & $0.24(0.08-0.77)$ \\
\cline { 1 - 2 } & & $0.12(0.03-0.49)$ & $0.16(0.05-0.49)$ & $0.13(0.03-0.52)$ \\
\hline
\end{tabular}

Gemcitabine was referent in all models. AHR is adjusted by year of diagnosis, weeks of neoadjuvant chemotherapy, and type of neoadjuvant chemotherapy. HR, hazard ratio; CAPE, capecitabine; GEM, gemcitabine; AHR, adjusted hazard ratio; $\mathrm{Cl}$, confidence interval.
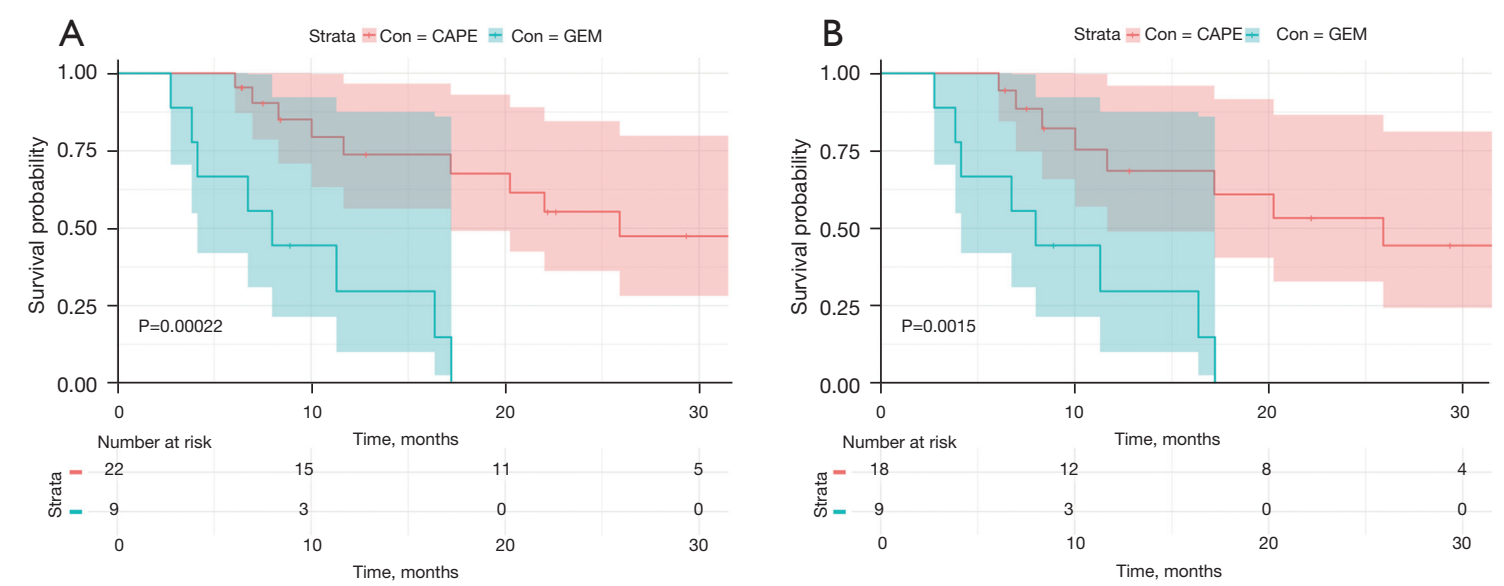

Figure 2 Kaplan-Meier plot of OS for chemoradiation with concurrent CAPE $v$ s. GEM with (A) or without (B) resectable patients included in analysis. OS, overall survival; CAPE, capecitabine; GEM, gemcitabine.

\section{OS}

Patients treated with CAPE-based 3 WCRT had a statistically superior mOS of 25.9 (95\% CI: 11.7-71.6), whereas the GEM group had a mOS of 8.0 months (95\% CI: 2.8-16.4), $\mathrm{P}<0.01$ (Figure 2A). This difference corresponded to a hazard ratio of 0.13 (95\% CI: 0.04-0.40) (Table 3). After excluding the resectable patients from the CAPE group this effect persisted: the CAPE group had a mOS of 25.9 (95\% CI: 10.0-71.6), $\mathrm{P}<0.01$ (Figure $2 B$ ). This difference corresponded to a hazard ratio of 0.16 (95\% CI: 0.05-0.49) (Table 3). After adjusting for relevant covariates using multivariable Cox regression, the CAPE group still had lower hazards of death than the GEM group (AHR $=0.12$; 95\% CI: 0.03-0.49) (Table 3). This effect persisted even after excluding the resectable patients at diagnosis $(\mathrm{AHR}=0.13 ; 95 \%$ CI: 0.03-0.52) (Table 3).

Sensitivity analyses excluding patients who were not treated with neoadjuvant FOLIFIRINOX were also conducted and results were summarized in the Table S2. Of note, the CAPE group still had a strong trend toward superior survival over the GEM group when analyzing borderline resectable and resectable patients $(\mathrm{HR}=0.32$; 95\% CI: 0.08-1.31). These effects were similar when excluding resectable patients and controlling for confounding with multivariable Cox regression.

\section{Patterns of progression}

Among 17 patients evaluable for progression (12 in the CAPE group and 5 in the GEM group), 2 (17\%) and 1 (20\%) patient first progressed only locally in the pancreas in the CAPE and GEM group, respectively. There were $7(58 \%)$ patients with distant only first progression in the CAPE group compared to 4 (80\%) in the GEM group. The patterns of first progression after 3 WCRT are summarized in Table 4. 
Table 4 Patterns of progression after short-course chemoradiotherapy with concurrent CAPE or GEM

\begin{tabular}{lcc}
\hline $\begin{array}{l}\text { Location of } \\
\text { progression }\end{array}$ & $\begin{array}{c}\text { CAPE-based, } \\
\mathrm{n}=12, \mathrm{n}[\%]\end{array}$ & $\begin{array}{c}\text { GEM-based, } \\
\mathrm{n}=5, \mathrm{n}[\%]\end{array}$ \\
\hline Local & $2[16.7]$ & $1[20.0]$ \\
Regional & $2[16.7]$ & $0[0.0]$ \\
Distant & $7[58.3]$ & $4[80.0]$ \\
Local and distant & $1[8.3]$ & $0[0.0]$ \\
\hline
\end{tabular}

CAPE, capecitabine; GEM, gemcitabine.

\section{Discussion}

This retrospective study compares CAPE and GEM concurrent chemotherapy in patients receiving a 3-week regimen of chemoradiation. Our primary objectives were to describe the odds of achieving surgical resection after neoadjuvant treatment and compare TTP of patients treated with 3WCRT with GEM-based or CAPE-based concurrent chemotherapy. Patients treated with CAPE-based regimens demonstrated higher odds of achieving surgical resection when compared to patients treated with concurrent GEM. Also, TTP was significantly superior in the CAPE group in univariable and multivariable analysis. Although not a primary aim of this study, we also observed superior OS in the CAPE group in univariable and multivariable models. These results are consistent with the data from the phase II SCALOP trial demonstrating superiority of concurrent CAPE with long-course chemoradiotherapy (50.4 Gy in 28 fractions) (14). Therefore, concurrent CAPE may have superior synergy with CRT including 3 WCRT.

The most compelling evidence supporting the use of 3 WCRT in (B)RPC stems from the Dutch PREOPANC trials $(13,15)$. The phase III PREOPANC trial supported the superiority of 3 WCRT with concurrent GEM over immediately proceeding to surgery, with an R0 resection rate of $71 \%$ vs. $40 \%$ in the 3 WCRT and immediate surgery arms, respectively. Our study demonstrated a much lower rate of resection in the GEM group (22.2\%). These differences could be explained by the higher proportion of resectable patients included in the PREOPANC trial and the greater heterogeneity in patients treated outside of the context of a trial.

Another study examining the relative efficacy of concurrent chemotherapy approaches in PDAC was performed in definitive setting for patients with locally advanced pancreatic cancer (16). The investigators tested concurrent GEM $\left(600 \mathrm{mg} / \mathrm{m}^{2} / \mathrm{kg}\right.$ for 6 weeks $) v s$. 5 -flourouracil $\left(500 \mathrm{mg} / \mathrm{m}^{2} /\right.$ day for days $1-3$, days $15-17$, and days 29-31) with long-course chemoradiation (50.4-61.2 Gy in 1.8 Gy fractions). The study demonstrated that at this dose, concurrent GEM was more effective than 5 -flurouracil but had a trend for more events of grade 3 and 4 neutropenia. A subsequent meta-analysis comparing GEM with 5-fluorouracil in LAPC concluded there were superior 12-month survival rates in GEM treated patients ( $\mathrm{RR}=1.54 ; 95 \%$ CI: 1.05-2.26) (17). Although these studies compared GEM with 5-fluourouracil and not CAPE and were conducted in the locally advanced instead of (B)RPC setting, these studies suggested GEM may be the superior radiosensitizer for treating patients with PDAC. It is possible that GEM's superiority over 5-fluorouracil is not at odds with our findings of the superiority of CAPE. Relative survival benefit of a concurrent chemotherapy could be due to fewer treatment breaks and dose-reductions along with superior radiosensitization. CAPE, the oral pro-drug of 5 -fluourouracil, may enable better tolerability leading to superior outcomes.

In the setting of (B)RPC, the phase II SCALOP trial was the first to demonstrate that choice of concurrent chemotherapy may influence the overall effectives of chemoradiation $(14,18)$. In contrast to the studies of GEM and 5 -fluorouracil in locally advanced pancreatic cancer, the SCALOP trial demonstrated superiority of CAPE over GEM. Our study also adds to this body of literature. In our study, 3WCRT with concurrent CAPE had a TTP of 15.42 months, similar to the progression free survival in the SCALOP trial of 12.0 months. The unadjusted and adjusted hazards ratios for progression in our study of 0.33 (95\% CI: $0.14-0.81$ ) and 0.24 (95\% CI: $0.08-0.77$ ) were analogous to what the HR observed in the SCALOP trial. Interestingly, the adjusted HR for progression in the SCALOP trial were not significant but were significant in our analysis. However, both OS estimates showed benefit of CAPE. 3WCRT with concurrent CAPE had improved survival outcomes over 3WCRT with concurrent GEM (HR $=0.13 ;$ 95\% CI: 0.04-0.40) even after restricting the dataset to only borderline resectable patients ( $\mathrm{HR}=0.16$; 95\% CI: $0.05-0.49)$ and controlling for confounding factors (AHR $=0.13$; 95\% CI: 0.03-0.52). These same trends were also seen in the SCALOP trial in unadjusted $(\mathrm{HR}=0.50$; 95\% CI: $0.27-0.93)$ and adjusted analyses $(\mathrm{HR}=0.39$; 95\% CI: 0.18-0.81). One critique of the SCALOP trial is that the investigators compared a relatively low dose of concurrent GEM (weekly infusions of $300 \mathrm{mg} / \mathrm{m}^{2}$ ) to full 
dose CAPE $\left(830 \mathrm{mg} / \mathrm{m}^{2}\right.$, twice daily on radiation treatment days). Our study did not have this same limitation; patients receiving GEM received a median dose of $675 \mathrm{mg} / \mathrm{m}^{2}$ (IQR: $600-912.5$ ), and $91 \%$ of patients received full dose CAPE. 3WCRT enables a convenient treatment schedule with highly effective concurrent chemotherapy, and our data suggest that concurrent CAPE may be superior to concurrent GEM when employing this approach.

Our findings are meaningful in the context of studies demonstrating the lack of benefit of chemoradiation relative to chemotherapy alone (19). Variability of fractionation and concurrent chemotherapy limits broad stroke conclusions about the utility of RT. For example, the much-anticipated PREOPANC-2 trial (TrialRegister.nl: NTR7292) testing 3WCRT with GEM vs. FOLFIRINOX alone and PANDAS-PRODIGE 44 (ClinicalTrials.gov: NCT02676349) trial testing a longer course of chemoradiation (50.4 Gy in 28 fractions) with CAPE $v s$. FOLFIRINOX alone are testing distinct fractionation and concurrent chemotherapeutic approaches. Negative outcomes from either trial could be attributed to the fractionation (3-vs. 6-week) or the concurrent chemotherapeutic strategy (GEM vs. CAPE). One could hypothesize that the PREOPANC-2 protocol might benefit from concurrent CAPE over concurrent GEM.

A major limitation of this analysis was its retrospective nature and the potential for confounding. A higher proportion of patients who were treated with concurrent CAPE were also treated with neoadjuvant FOLFIRNIOX. Despite including this covariate in multivariable analyses, residual confounding may persist. We aimed to address this limitation by running a sensitivity analysis excluding patients not treated with neoadjuvant FOLFIRINOX in both the GEM and CAPE groups. In this small subsample, statistical significance was not observed, and point estimates for survival were attenuated. Because of the limited sample size of our study, we were only able to include a few covariates in our models; residual measured and unmeasured confounding could have also biased the surgical and survival outcomes measured. For example, the patients treated with GEM may have had more advanced disease that was less amenable to resection or a poorer performance status. It should also be noted that the first studies evaluating 3WCRT utilized full-dose GEM (weekly infusions of $\left.1,000 \mathrm{mg} / \mathrm{m}^{2}\right)(10,12)$; the 3 WCRT regimen aimed to shrink treatment volumes and omit elective nodes while delivering highly effective chemotherapy treatment. However, the median GEM dose delivered in our study was weekly infusions of $675 \mathrm{mg} / \mathrm{m}^{2}$, which may have been insufficient to match the effectiveness of the twice daily $825 \mathrm{mg} / \mathrm{m}^{2}$ CAPE. It is possible that if patients had received higher doses of concurrent GEM, they would have had superior surgical and survival outcomes. Additionally, the treatments after chemoradiation may have differed significantly between patients in the GEM and CAPE groups, especially because the long eligibility period. While TTP advantages of CAPE-based treatments may have been accurate estimates, the high heterogeneity in post-3WCRT management of these patients limits the generalizability and importance of the mOS endpoint in determining relative effectiveness of these regimens. For this reason, we believe this study should be interpreted as hypothesis generating, and we call for additional studies to definitively test these hypotheses.

\section{Conclusions}

Patients diagnosed with (B)RPC have many therapeutic options. This retrospective study suggests a benefit to CAPE-based concurrent chemotherapy when treating patients with 3 WCRT, consistent with prior literature of long-course chemoradiotherapy. While 3WCRT for the management of patients with (B)RPC may lose popularity as ablative treatments become more ubiquitous, carefully choosing a concurrent chemotherapy remains an important decision for the multidisciplinary team. Not all concurrent regimens should be treated equally, and randomized studies are needed to definitively examine if CAPE-based chemoradiation is the optimal treatment.

\section{Acknowledgments}

Part of this work was supported by Chancellor's Global Health Scholarship from Rutgers Biomedical and Health Sciences.

Funding: Research reported in this publication was supported by the National Center for Advancing Translational Sciences (NCATS), a component of the National Institutes of Health (NIH) under award number UL1TR003017 (SSN). The content is solely the responsibility of the authors and does not necessarily represent the official views of the NIH.

\section{Footnote}

Reporting Checklist: The authors have completed the STROBE reporting checklist. Available at https://dx.doi. 
org/10.21037/jgo-21-503

Data Sharing Statement: Available at https://dx.doi. org/10.21037/jgo-21-503

Conflicts of Interest: All authors have completed the ICMJE uniform disclosure form (available at https://dx.doi. org/10.21037/jgo-21-503). The authors have no conflicts of interest to declare.

Ethical Statement: The authors are accountable for all aspects of the work in ensuring that questions related to the accuracy or integrity of any part of the work are appropriately investigated and resolved. The study was conducted in accordance with the Declaration of Helsinki (as revised in 2013). The study was approved by institutional ethics board of Rutgers Biomedical and Health Sciences (No. Pro2018002435) and individual consent for this retrospective analysis was waived.

Open Access Statement: This is an Open Access article distributed in accordance with the Creative Commons Attribution-NonCommercial-NoDerivs 4.0 International License (CC BY-NC-ND 4.0), which permits the noncommercial replication and distribution of the article with the strict proviso that no changes or edits are made and the original work is properly cited (including links to both the formal publication through the relevant DOI and the license). See: https://creativecommons.org/licenses/by-nc-nd/4.0/.

\section{References}

1. American Cancer Society. Cancer Facts and Figures 2020. Atlanta, GA, USA: American Cancer Society, 2020.

2. Li D, Xie K, Wolff R, et al. Pancreatic cancer. Lancet 2004;363:1049-57.

3. Versteijne E, Vogel JA, Besselink MG, et al. Meta-analysis comparing upfront surgery with neoadjuvant treatment in patients with resectable or borderline resectable pancreatic cancer. Br J Surg 2018;105:946-58.

4. Dhir M, Malhotra GK, Sohal DPS, et al. Neoadjuvant treatment of pancreatic adenocarcinoma: a systematic review and meta-analysis of 5520 patients. World J Surg Oncol 2017;15:183.

5. Janssen QP, Buettner S, Suker M, et al. Neoadjuvant FOLFIRINOX in Patients With Borderline Resectable Pancreatic Cancer: A Systematic Review and Patient-Level Meta-Analysis. J Natl Cancer Inst 2019;111:782-94.
6. Gillen S, Schuster T, Meyer Zum Büschenfelde C, et al. Preoperative/neoadjuvant therapy in pancreatic cancer: a systematic review and meta-analysis of response and resection percentages. PLoS Med 2010;7:e1000267.

7. Sohn TA, Yeo CJ, Cameron JL, et al. Resected adenocarcinoma of the pancreas-616 patients: results, outcomes, and prognostic indicators. J Gastrointest Surg 2000;4:567-79.

8. Palta M, Godfrey D, Goodman KA, et al. Radiation Therapy for Pancreatic Cancer: Executive Summary of an ASTRO Clinical Practice Guideline. Pract Radiat Oncol 2019;9:322-32.

9. McGinn CJ, Zalupski MM, Shureiqi I, et al. Phase I trial of radiation dose escalation with concurrent weekly fulldose gemcitabine in patients with advanced pancreatic cancer. J Clin Oncol 2001;19:4202-8.

10. Talamonti MS, Small W Jr, Mulcahy MF, et al. A multiinstitutional phase II trial of preoperative full-dose gemcitabine and concurrent radiation for patients with potentially resectable pancreatic carcinoma. Ann Surg Oncol 2006;13:150-8.

11. Murphy JD, Adusumilli S, Griffith KA, et al. Full-dose gemcitabine and concurrent radiotherapy for unresectable pancreatic cancer. Int J Radiat Oncol Biol Phys 2007;68:801-8.

12. Small W Jr, Berlin J, Freedman GM, et al. Full-dose gemcitabine with concurrent radiation therapy in patients with nonmetastatic pancreatic cancer: a multicenter phase II trial. J Clin Oncol 2008;26:942-7.

13. Versteijne E, Suker M, Groothuis K, et al. Preoperative Chemoradiotherapy Versus Immediate Surgery for Resectable and Borderline Resectable Pancreatic Cancer: Results of the Dutch Randomized Phase III PREOPANC Trial. J Clin Oncol 2020;38:1763-73.

14. Mukherjee S, Hurt CN, Bridgewater J, et al. Gemcitabinebased or capecitabine-based chemoradiotherapy for locally advanced pancreatic cancer (SCALOP): a multicentre, randomised, phase 2 trial. Lancet Oncol 2013;14:317-26.

15. Janssen QP, Homs MYV, Wilmink JW, et al. Neoadjuvant FOLFIRINOX versus neoadjuvant chemoradiotherapy and adjuvant chemotherapy for (borderline) resectable pancreatic cancer: update on the PREOPANC-2 study. HPB 2020;22:S277.

16. Li CP, Chao Y, Chi KH, et al. Concurrent chemoradiotherapy treatment of locally advanced pancreatic cancer: gemcitabine versus 5 -fluorouracil, a randomized controlled study. Int J Radiat Oncol Biol Phys 2003;57:98-104. 
17. Zhu CP, Shi J, Chen YX, et al. Gemcitabine in the chemoradiotherapy for locally advanced pancreatic cancer: a meta-analysis. Radiother Oncol 2011;99:108-13.

18. Hurt CN, Falk S, Crosby T, et al. Long-term results and recurrence patterns from SCALOP: a phase II randomised trial of gemcitabine- or capecitabine-based chemoradiation for locally advanced pancreatic cancer. Br J Cancer

Cite this article as: Neibart SS, Mamidanna S, Chundury A, Sayan M, Alexander HR, August DA, Berim LD, Boland PM, Grandhi MS, Gulhati P, Hochster HS, Langan RC, Spencer KR, Kennedy TJ, Deek MP, Jabbour SK. Outcomes of patients with borderline resectable and resectable pancreatic adenocarcinoma treated with neoadjuvant three-week course chemoradiotherapy using capecitabine-based versus gemcitabine-based concurrent chemotherapy. J Gastrointest Oncol 2021;12(6):2557-2566. doi: 10.21037/jgo-21-503
2017;116:1264-70.

19. Katz MHG, Shi Q, Meyers JP, et al. Alliance A021501: Preoperative mFOLFIRINOX or mFOLFIRINOX plus hypofractionated radiation therapy (RT) for borderline resectable (BR) adenocarcinoma of the pancreas. J Clin Oncol 2021;39:377. 


\section{Supplementary}

Table S1 Sensitivity analysis excluding patients not treated with neoadjuvant FOLFIRINOX-HR of progression for patients treated with chemoradiation with concurrent CAPE or GEM with or without resectable patients in analysis

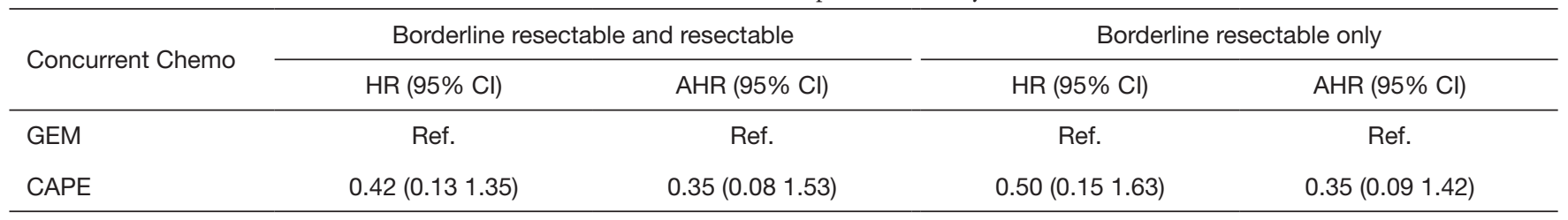

AHR is adjusted by year of diagnosis, weeks of neoadjuvant chemotherapy, and type of neoadjuvant chemotherapy. HR, hazard ratio; CAPE, capecitabine; GEM, gemcitabine; AHR, adjusted hazard ratio; $\mathrm{Cl}$, confidence interval; Chemo, chemotherapy.

Table S2 Sensitivity analysis excluding patients not treated with neoadjuvant FOLFIRINOX_HR of death for patients treated with chemoradiation with concurrent Xeloda or GEM with or without resectable patients in analysis

\begin{tabular}{lccccc}
\hline \multirow{2}{*}{ Concurrent Chemo } & \multicolumn{2}{c}{ Borderline resectable and resectable } & & \multicolumn{2}{c}{ Borderline resectable Only } \\
\cline { 2 - 3 } \cline { 5 - 6 } GEM & $\mathrm{HR}(95 \% \mathrm{Cl})$ & $\mathrm{AHR}(95 \% \mathrm{Cl})$ & $\mathrm{HR}(95 \% \mathrm{Cl})$ & $\mathrm{AHR}(95 \% \mathrm{Cl})$ \\
CAPE & Ref. & Ref. & Ref. & Ref. \\
\hline
\end{tabular}

AHR is adjusted by year of diagnosis, weeks of neoadjuvant chemotherapy, and type of neoadjuvant chemotherapy. HR, hazard ratio; GEM, gemcitabine; AHR, adjusted hazard ratio; $\mathrm{Cl}$, confidence interval; Chemo, chemotherapy. 